\title{
Septal Deviation
}

National Cancer Institute

\section{Source}

National Cancer Institute. Septal Deviation. NCI Thesaurus. Code C120906.

An alteration of the septum from the midline. 